\title{
Maxwell Nanofluid Flow over an Infinite Vertical Plate with Ramped and Isothermal Wall Temperature and Concentration
}

\author{
Naveed Khan $\left(\mathbb{D},{ }^{1}\right.$ Farhad Ali $\left(\mathbb{D},{ }^{1}\right.$ Muhammad Arif $\mathbb{D}^{1},{ }^{1}$ Zubair Ahmad $\left(\mathbb{D},{ }^{1}\right.$ \\ Aamina Aamina $\mathbb{D}^{2,3}$ and Ilyas Khan $\mathbb{D}^{4}$ \\ ${ }^{1}$ Department of Mathematics, City University of Science and Information Technology, Peshawar 25000, Khyber \\ Pakhtunkhwa, Pakistan \\ ${ }^{2}$ Computational Analysis Research Group, Ton Duc Thang University, Ho Chi Minh City 70000, Vietnam \\ ${ }^{3}$ Faculty of Mathematics and Statistics, Ton Duc Thang University, Ho Chi Minh City 70000, Vietnam \\ ${ }^{4}$ Department of Mathematics, College of Science Al-Zulf, Majmaah University, Al-Majmaah 11952, Saudi Arabia
}

Correspondence should be addressed to Aamina Aamina; aamina@tdtu.edu.vn

Received 12 April 2021; Revised 16 July 2021; Accepted 29 July 2021; Published 7 September 2021

Academic Editor: Constantin Fetecau

Copyright @ 2021 Naveed Khan et al. This is an open access article distributed under the Creative Commons Attribution License, which permits unrestricted use, distribution, and reproduction in any medium, provided the original work is properly cited.

\begin{abstract}
The aim of this study is to investigate how heat and mass transfer impacts the unsteady incompressible flow of Maxwell fluid. An infinite vertical plate with ramped and isothermal wall temperature and concentration boundary conditions is considered with the Maxwell fluid. Furthermore, in this study, engine oil has been taken as a base fluid due to its enormous applications in modern science and technologies. To see the importance of nanofluids, we have suspended molybdenum disulfide in engine oil base fluid to enhance its heat transfer rate. To investigate the flow regime, the system of equations was derived in the form of partial differential equations. The exact solutions to the complex system are obtained using the Laplace transform technique. Graphically, the impact of different embedded parameters on velocity, temperature, and concentration distributions has been shown. Through using the graphical analysis, we were interested in comparing the velocity, temperature, and concentration profiles for ramped and isothermal wall temperature and concentration. The magnitude of velocity, temperature, and concentration distributions is greater for an isothermal wall and less for a ramped wall, according to our observations. We observed that adding molybdenum disulfide nanoparticles to the engine oil increased the heat transfer up to $12.899 \%$. Finally, the corresponding skin friction, Nusselt number, and Sherwood number have been calculated and presented in a tabular form.
\end{abstract}

\section{Introduction}

There are several types of fluids that cannot be described by simple Navier-Stokes equations because of their complex rheology. These fluids are called non-Newtonian fluids which have many applications in our daily life such as biotechnologies, geophysics, astrophysics, industries, and engineering developments. Therefore, researchers are attracted to study the non-Newtonian fluids. Non-Newtonian fluids may be further classified as differential, rate, and integral type fluids. Integral type fluids are those in which the actual Cauchy stress is calculated by reading an integral over the background of the general deformation gradient. Differential type fluids are those of which the stress is determined by the velocity inclination and its various higher time derivatives. As movement ceases in different rates compressible fluids, the stress reduces to an uncertain circular stress. In comparison to differential type fluids, where the stress is distributed unequally as a function of the velocity gradient and its higher time derivatives, rate type liquid models have an implied relationship between the stress and its higher time derivatives. The Maxwell fluid is a viscoelastic fluid of rate type that relates to the non-Newtonian fluid class. In terms of experimentation, rate type models are more realistic since they account for both memory and elastic effects [1]. As a consequence, the Maxwell model, a subclass of rate type models, has been included in the study. The concept of Maxwell fluid was 
given by Maxwell [2]. In the physical situation, many fluids such as engine oil (EO), glycerin, and some other plyometric materials behave like Maxwell fluid. Many researchers have considered Maxwell fluid in their studies like Maxwell nanofluid flow over a vertical plate was analyzed by Aman et al. [3] using the Laplace transform technique to find accurate solution to partial differential equations for initial and boundary conditions. Arif et al. [4] studied Maxwell nanofluid flow and discussed some applications of their results in $\mathrm{EO}$ with the influence of ramped wall conditions. Khalid et al. [5] calculated the results of ferrofluid in a permeable channel along a vibrating plate with ramped and isothermal wall temperature (RWT and IWT). Zhao et al. [6] reported the results of Maxwell nanofluid flow with the influence of heat and mass transfer with Dufour influences using the Caputo fractional model. The characteristics of wave motion occurring in the Maxwell nanofluid rheometer was studied by Huilgol [7]. Jamil [8] calculated the Maxwell nanofluid flow along with a vibrating plate, the effect of shear stress with no-slip condition using discreet Laplace transform to find the closed form of solutions. Convective flow of a nonsteady Maxwell nanofluid over an infinite vertical plate with ramped conditions was examined by Anwar et al. [9]. $\mathrm{Na}$ et al. [10] analyzed free convection Maxwell nanofluid flow between vertical plates with the influence of thermal radiation. Raza and Asad [11] derived the solutions of Maxwell fluid flow considering a vertical flat plate. They observed that increasing the fluid velocity with respect to Grashof number (Gr) and Maxwell parameter $\lambda$. The numerical solutions were obtained by Sui et al. [12] calculating the Maxwell nanofluid over stretching sheet with the influence of heat and mass transfer using the homotopy analysis process. Wang and Tan [13] discussed the Maxwell nanofluid flow passing through a permeable channel with the Soret effect.

Heat is a form of energy that can be moved from higher concentration to a lower concentration. Heat can be transfered through radiation, conduction, and convection. Transfer of heat has many applications in industrial and manufacturing processes like cooling chambers, heating phenomena, engines, and drying processes. Similarly, the net movement from one region to another is known as mass transfer. Distillation, filtration, crystallization, precipitation, evaporation, and absorption are only some of the processes that include mass transfer. It has wide applications in modern sciences and technologies like purification of crude oil, petrochemical refining, fractional distillation, highpressure pumps, and refrigeration. Steeman et al. [14] analyzed mass and heat measurement for water vaporization. The effects of heat and mass transfer study of water and gas for waste heat recovery were investigated by Men et al. [15]. Chakkinga et al. [16] studied the heat and mass transfer in adiabatic cylindrical obstacles. Yan et al. [17] examined planar layer for fuel cell considering mass and heat transfer. Chu et al. [18] studied the physical characteristics of ammonia regeneration with mass and heat transfer. Hazarika et al. [19] calculated that fork's usages influenced the fin preparation project for instantaneous mass and heat transfer in a space-controlled position. Khan et al. [20] examined intermittent microwave convective drying modelling of the combined influence of heat and mass. Luikov [21] analyzed the fluid flow in a capillary absorbent organ with the mutual influence of mass and heat. Riaz et al. [22] described Maxwell nanofluid flow by taking the effect of heat and mass transfer. Chen et al. [23] analyzed the experimental study of the mass and heat properties of water and air in an elliptical plane tube.

The ramped temperature on the fluid is a temperature variation during a given time period. The ramped or nonuniform wall temperature has many applications in modern sciences and technologies such as engineering, industrial, and several other physical applications such as automobile, electric circuit, heat transfer in the building, solar collector, nuclear energy, geothermal, air condition, and photovoltaic mechanism. Similarly, isothermal temperature means constant temperature. Generally, it describes a thermodynamic process where the temperature is constant. It has many modern science and technologies applications such as the factory automation system, robot's system, water handling system, automobiles, airplanes and boats, vehicles, mechanical structure, electrical power, plants, animals, transpiration, and computers. Chandran et al. [24] calculated the natural convection fluid flow by taking RWT near the plate. Khalid et al. [25] discussed the free convection nanofluids with RWT. The transient free convection fluid flow with RWT is analyzed by Narahari [26]. RWT conditions as given by Ghara et al. [27] were used to investigate the impact of magnetohydrodynamic (MHD) free convection flow over a fluctuating layer. RWT and slip conditions investigated by Haq et al. [28] were used to the unsteady viscous fluid flow. Some other real-world and industrial applications of ramped and isothermal wall concentration and temperature like generator systems, biofluids, blood flow, and nanofluids can be found in $[9,29-34]$.

Heat and mass transfer have many physical applications in today's sciences and technology. The thermal conductivity of traditional fluids such as ethylene glycol, water, polyethylene glycol, and EO is poor. To improve the thermal conductivity of their materials, Maxwell [35] developed the concept of microparticle dispersion in regular fluids. Still, there were few restrictions in the Maxwell model because these particles are very heavy and have very quick settled down, clogging of narrow channels, and cause destruction of these channels. To overcome this issue, Choi [36] developed a new concept for interrupting nanosized particles in regular fluids to improve thermal conductivity. In a rotating frame, the Jeffery nanofluid flow was examined by Ali et al. [37] with the impact of heat transfer analysis. They observed that the heat will enhance up to $12.37 \%$ by adding silver nanoparticles from 0.00 to 0.04 in the base fluid EO. Using EO as a base fluid, the Brinkman-type fluid flow with spherical form molybdenum disulfide $\left(\mathrm{MoS}_{2}\right)$ nanoparticles 
was investigated Jan et al. [38]. Some applications of nanoparticles in different regular fluids are discussed in [39-42]. Ali et al. [43] examined the applications of EO in modern sciences, taking molybdenum disulfide $\mathrm{MoS}_{2}$ nanoparticles in it. The applications of different nanoparticles to improve the heat transfer rate are discussed by many researchers $[44,45]$. Turkyilmazoglu [46] studied the flow past a vertical infinite isothermal plate in a viscous electrically conducting natural convective incompressible nanofluid. They take into account the effects of heat absorption, heat generation, and radiation. Akinshilo et al. [47] investigated the steady incompressible flow of a nonNewtonian sodium alginate (SA) fluid conveying copper nanoparticles $(\mathrm{Cu})$ which flow within two vertical parallel plates. The obtained results can be used off the sodium alginate in processes such as manufacturing and biomedical applications. Turkyilmazoglu [48] analyzed the phenomenon of interaction of suspended particles within the fluid over a stretchable rotating disk. They observed that the dust phase has reduced velocity and temperature as compared to those of the fluid phase. The heat and mass transfer of a radiating non-Newtonian sodium alginate transported through parallel squeezing plates was examined by Akinshilo et al. [49] Turkyilmazoglu [50] analyzed the flow and heat transfer of the nanofluid film flow over a moving inclined substrate. They observed that the motion of nanoparticles is induced by the action of both the gravitational force as well as the substrate movement. Akinshilo and Sobamowo [51] investigated the flow of blood with gold nanoparticles equally suspended through a porous channel. They developed models to show the effects of the nanoparticles on the concentration, temperature, and velocity of the fluid as it flows through the porous medium.

Based on the abovementioned literature, no work has been performed to obtain Maxwell nanofluid close form solutions with the combined effect of heat and mass transfer. We also focused at how ramped and isothermal wall temperatures and concentrations influence the results. Furthermore, contributing to its wide physical and mechanical engineering applications, EO is used as a base fluid. To increase thermal conductivity, spherical shaped $\mathrm{MoS}_{2}$ nanoparticles have been spread in EO.

\section{Mathematical Formulation}

The unsteady incompressible Maxwell fluid flow over an infinite vertical plate with ramped and isothermal wall temperature and concentration was investigated in this research. $\mathrm{MoS}_{2}$ nanoparticles have been suspended uniformly in the base fluid EO to enhance the rate of heat transfer. The plate is taken along the $x$-axis, while the fluid occupies the space $(y \geq 0)$. Both the plate and the fluid are initially at rest with ambient temperature. $T_{1 \infty}$ and constant concentration $C_{1 \infty}$. After some time $t=0^{+}$, the temperature and concentration of the plate rise slowly to $T_{1 w}$ and $C_{1 w}$, respectively. In other words, at time $t=0^{+}$, temperature of the plate and concentration become higher or lower to $T_{1}+$ $\left(T_{1 w}-T_{1 \infty}\right) t_{1} / t_{0}$ and $C_{1 \infty}+\left(C_{1 w}-C_{1 \infty}\right) t_{1} / t_{0}$ when $t_{1} \leq t_{0}$, respectively. When $t_{1}>t_{0}$, no change will occur in concentration and temperature and remains at constant temperature and concentration. Keeping in mind the above assumptions, the governing equations for the above flow regime are derived [3].

For the above flow regime, the velocity distribution for unidirectional flow is defined as

$$
\vec{V}=(u(y, t), 0,0)
$$

Continuity equation is given as

$$
\nabla \cdot \vec{V}=0
$$

Using equations (1) and (2) satisfies. Using equation (1), the governing equation for the above flow regime is given as

$$
\begin{aligned}
\rho_{n f}\left(1+\lambda_{1} \frac{\partial}{\partial t_{1}}\right) \frac{\partial u_{1}\left(y_{1}, t_{1}\right)}{\partial t_{1}}= & \mu_{n f} \frac{\partial^{2} u_{1}\left(y_{1}, t_{1}\right)}{\partial y_{1}^{2}}+\left(1+\lambda_{1} \frac{\partial}{\partial t_{1}}\right)\left(\rho \beta_{T}\right)_{n f} g\left(T_{1}\left(y_{1}, t_{1}\right)-T_{1 \infty}\right) \\
& +\left(1+\lambda_{1} \frac{\partial}{\partial t_{1}}\right)\left(\rho \beta_{C}\right)_{n f} g\left(C_{1}\left(y_{1}, t_{1}\right)-C_{1 \infty}\right),
\end{aligned}
$$

and temperature equation is 


$$
\begin{aligned}
\left(\rho C_{p}\right)_{n f} \frac{\partial T_{1}}{\partial t_{1}} & =k_{n f} \frac{\partial^{2} T_{1}}{\partial y_{1}{ }^{2}}, \\
\frac{\partial C_{1}}{\partial t_{1}} & =D_{n f} \frac{\partial^{2} C_{1}}{\partial y_{1}{ }^{2}},
\end{aligned}
$$

subject to the following imposed physical condition.

$$
\begin{aligned}
& u_{1}(y, 0)=0,\left.\frac{\partial u_{1}(y, t)}{\partial t}\right|_{t=0}=0, \\
& T_{1}(y, 0)=T_{1 w}, \\
& C_{1}(y, 0)=C_{1 w}, \quad \text { for } t=0 \text { and } y \geq 0, \\
& u_{1}(0, t)=0, T_{1}(0, t)= \begin{cases}T_{1}+\left(T_{1 w}-T_{1 \infty}\right) \frac{t_{1}}{t_{0}}, & 0<t_{1} \leq t_{0}, \\
T_{1 w}, & t_{1} \geq t_{0},\end{cases} \\
& C_{1}(0, t)= \begin{cases}C_{1 \infty}+\left(C_{1 w}-C_{1 \infty}\right) \frac{t_{1}}{t_{0}}, & 0<t_{1} \leq t_{0}, \\
C_{1 w}, & \text { for } t>0 \text { and } y=0,\end{cases} \\
& u_{1}(\infty, t)=0, \\
& T_{1}(\infty, t)=T_{1 \infty},
\end{aligned}
$$

In the above system of equations, the velocity component of Maxwell nanofluid along the axis is $u_{1}, T_{1}$ represents the temperature distribution, $T_{1 w}$ is the wall temperature which is fixed, and $g$ is the gravitational acceleration. The thermophysical properties of the EO and $\mathrm{MoS}_{2}$ nanoparticles are given in Table 1.

$$
\begin{aligned}
& \rho_{n f}=\rho_{f}(1-\phi)+\phi \rho_{s}, \\
& \left(\rho \beta_{T}\right)_{n f}=\left(\rho \beta_{T}\right)_{f}(1-\phi)+\phi\left(\rho \beta_{T}\right)_{s}, \\
& \left(\rho \beta_{C}\right)_{n f}=\phi\left(\rho \beta_{C}\right)_{s}+\left(\rho \beta_{C}\right)_{f}(1-\phi), \\
& \left(\rho c_{p}\right)_{n f}=(1-\phi)\left(\rho c_{p}\right)_{f}+\phi\left(\rho c_{p}\right)_{s} \\
& D_{n f}=D_{f}(1-\phi), \\
& k_{n f}=k_{f}\left[\frac{k_{s}+2 k_{f}-2 \phi\left(k_{f}-k_{s}\right)}{k_{s}+2 k_{f}+\phi\left(k_{f}-k_{s}\right)}\right], \\
& \mu_{n f}=\mu_{f}(1-\phi)^{-2.5}
\end{aligned}
$$

$\mu_{n f}$ and $\mu_{f}$ represent the dynamic viscosity of the nanofluid and based fluid, respectively. Here, $\phi$ used to represent the volume fraction [43]. The following dimensionless variables were added to make the above system of equations dimensionless:

$$
\left.\begin{array}{l}
w=\frac{u_{1}}{U_{0}}, \\
\zeta=\frac{y_{1}}{\sqrt{v \tau}}, \\
\tau=\frac{t_{1}}{\tau_{0}}, \\
\tau_{0}=\left[\frac{v^{1 / 2}}{g \beta\left(T_{1 w}-T_{1 \infty}\right)}\right]^{2 / 3}, \\
\Theta=\frac{T_{1}-T_{1 \infty}}{T_{1 w}-T_{1 \infty}}, \\
\Phi=\frac{C_{1}-C_{1 \infty}}{C_{1 w}-C_{1 \infty}}
\end{array}\right\}
$$


TABLE 1: Thermophysical properties of EO and $\mathrm{MoS}_{2}$ [43]:

\begin{tabular}{lcccc}
\hline Material & $\rho\left(\mathrm{km}^{-3}\right)$ & $c_{p}\left(\mathrm{Jkg}^{-1} \mathrm{~K}^{-1}\right)$ & $k\left(\mathrm{wm}^{-1} \mathrm{~K}^{-1}\right)$ & \\
\hline Engine oil & 868 & 2048 & 0.1404 & 0.00007 \\
$\mathrm{MoS}_{2}$ & $506 \times 10^{3}$ & 397.21 & 904.4 & 2.8424 \\
\hline
\end{tabular}

Using equation (7), the momentum, temperature, and concentration equations nondimensionalized to the following form:

$$
\begin{aligned}
d\left(1+\lambda \frac{\partial}{\partial \tau}\right) \frac{\partial w(\zeta, \tau)}{\partial \tau} & =d_{1} \frac{\partial^{2} w(\zeta, \tau)}{\partial \zeta^{2}}+d_{2}\left(1+\lambda \frac{\partial}{\partial \tau}\right) \mathrm{Gr} \Theta+d_{3}\left(1+\lambda \frac{\partial}{\partial \tau}\right) \mathrm{Gm} \Phi \\
\operatorname{Pr} m_{3} \frac{\partial \Theta(\zeta, \tau)}{\partial \tau} & =\frac{\partial^{2} \Theta(\zeta, \tau)}{\partial \zeta^{2}} \\
\operatorname{Sc} \frac{\partial \Phi(\zeta, \tau)}{\partial \tau} & =(1-\phi) \frac{\partial^{2} \Phi(\zeta, \tau)}{\partial \zeta^{2}}
\end{aligned}
$$

Equations (8), (9), and (10) in more simplified form can be written as

$$
\begin{gathered}
\left(1+\lambda \frac{\partial}{\partial \tau}\right) \frac{\partial w(\zeta, \tau)}{\partial \tau}=m \frac{\partial^{2} w(\zeta, \tau)}{\partial \zeta^{2}}+m_{2}\left(1+\lambda \frac{\partial}{\partial \tau}\right) \operatorname{Gr} \Theta(\zeta, \tau)+m_{4}\left(1+\lambda \frac{\partial}{\partial \tau}\right) \operatorname{Gm} \Phi(\zeta, \tau), \\
\frac{\partial^{2} \Theta(\zeta, \tau)}{\partial \zeta^{2}}-b \frac{\partial \Theta(\zeta, \tau)}{\partial \tau}=0 \\
\frac{\partial^{2} \Phi(\zeta, \tau)}{\partial \zeta^{2}}-b_{1} \frac{\partial \Phi(\zeta, \tau)}{\partial \tau}=0 .
\end{gathered}
$$

With the dimensionless physical IC's and BCs are 


$$
\begin{aligned}
& w(\zeta, 0)=0, \\
& \left.\frac{\partial w(\zeta, \tau)}{\partial \tau}\right|_{\tau=0}=0 \\
& \Theta(\zeta, 0)=0, \\
& \Phi(\zeta, 0)=0, \\
& w(0, \tau)=0, \\
& \Theta(0, \tau)=\left\{\begin{array}{ll}
\tau, & 0<\tau \leq 1, \\
1, & \tau>1,
\end{array}=t H(\tau)-(\tau-1) H(\tau-1),\right\} \\
& \Phi(0, \tau)=\left\{\begin{array}{ll}
\tau, & 0<\tau \leq 1, \\
1, & \tau>1,
\end{array}=t H(\tau)-(\tau-1) H(\tau-1)\right. \\
& w(\infty, \tau)=0, \\
& \Theta(\infty, \tau)=0, \\
& \Phi(\infty, \tau)=0
\end{aligned}
$$

where $H(\tau)$ is the Heaviside step function, and some dimensionless numbers/constants which appear during calculations are given as follows.

$$
\begin{aligned}
\lambda & =\frac{\lambda_{1}}{\tau_{0}} \\
\mathrm{Gr} & =\frac{g_{x}\left(\beta_{T}\right)_{f}\left(T_{1 w}-T_{1 \infty}\right) \tau_{0}}{U_{0}}, \\
\operatorname{Pr} & =\frac{\left(\mu C_{p}\right)_{f}}{k_{f}} \\
m & =\frac{d}{d_{1}} \\
m_{1} & =\frac{d_{2}}{d_{1}} \\
m_{2} & =m_{1} \mathrm{Gr} \\
m_{3} & =\frac{d_{3}}{d_{4}} \\
b & =m_{3} \mathrm{Pr}
\end{aligned}
$$

$$
\begin{aligned}
\mathrm{Gm} & =\frac{g_{x}\left(\beta_{C}\right)_{f}\left(C_{1 w}-C_{1 \infty}\right) \tau_{0}}{U_{0}}, \\
b_{1} & =\frac{\mathrm{Sc}}{1-\phi}, \\
m_{4} & =m_{1} \mathrm{Gm}, \\
d & =(1-\phi)+\phi \frac{\rho_{s}}{\rho_{f}}, \\
d_{1} & =\frac{1}{(1-\phi)^{2.5}}, \\
d_{2} & =(1-\phi)+\phi \frac{\left(\rho \beta_{T}\right)_{s}}{\left(\rho \beta_{T}\right)_{f}}, \\
d_{3}= & (1-\phi)+\phi \frac{\left(\rho c_{p}\right)_{s}}{\left(\rho c_{p}\right)_{f}}, \\
d_{4}= & \frac{\left(k_{s}+2 k_{f}\right)-2 \phi\left(k_{f}-k_{s}\right)}{\left(k_{s}-2 k_{f}\right)+\left(k_{f}-k_{s}\right)}, \\
\mathrm{Sc} & =\frac{v_{f}}{D_{f}},
\end{aligned}
$$

where Pr denotes the Prandtl number, Gr and Gm denote the thermal and mass Grashof numbers, Sc represents the Schmidt number, and $\lambda$ represents the dimensionless Maxwell nanofluid parameter. 


\section{Solution of the Problem}

To find the solution of the dimensionless system, we first consider the energy equation.

3.1. Exact Solution of Energy Equation. We use the Laplace transform technique to solve equation (12), and incorporating the ICs and $\mathrm{BCs}$, we get

$$
\bar{\Theta}(\zeta, p)=\frac{1}{p^{2}} e^{-\zeta \sqrt{b p}}-\frac{e^{-p}}{p^{2}} e^{-\zeta \sqrt{b p}},
$$

inverting the Laplace transform, equation (16) for ramped wall temperature becomes

$$
\Theta(\zeta, \tau)_{\mathrm{ramp}}=\Theta_{1}(\zeta, \tau)-\Theta_{1}(\zeta, \tau-1) H(\tau-1) .
$$

3.1.1. Error Function. The error function equals twice the integral of a normalized Gaussian function between 0 and $x / \sigma \sqrt{2}$, where $\sigma$ is the standard distribution.

$$
y=\operatorname{erf}(x)=\frac{2}{\sqrt{\pi}} \int_{0}^{x} e^{-t^{2}} \mathrm{~d} t, \quad \text { for } x \geq 0, y[0,1]
$$

3.1.2. Complementary Error Function. In terms of error function, the complementary error function can be expressed as follows:

$$
1-y=\operatorname{erfc}(x)=1-\operatorname{erf}(x)=\frac{2}{\sqrt{\pi}} \int_{x}^{\infty} e^{-t^{2}} \mathrm{~d} t, \quad \text { for } x \geq 0, y[0,1]
$$

and solution for isothermal wall temperature becomes

where

$$
\Theta_{1}(\zeta, \tau)_{i s o}=\operatorname{erfc}\left[\frac{\zeta \sqrt{b}}{2 \sqrt{\tau}}\right]
$$

$$
\Theta_{1}(\zeta, \tau-1)=\left[\left(\operatorname{erfc}\left(\frac{\zeta}{2} \sqrt{\frac{b}{\tau}}\right)\right)\left(\tau+\frac{b \zeta^{2}}{2}\right)-\zeta \sqrt{b} \sqrt{\frac{\tau}{\pi} e^{-(b \zeta / 4 \tau)}}\right]
$$

3.2. Exact Solution of Concentration Equation. Using the Laplace transform technique to solve equation (13) and including ICs and BCs, we will find the solution to the concentration equation:

$$
\bar{\Phi}(\zeta, p)=\frac{1}{p^{2}} e^{-\zeta \sqrt{b_{1} p}}-\frac{e^{-p}}{p^{2}} e^{-\zeta \sqrt{b_{1} p}},
$$

and solution for isothermal wall concentration becomes

$$
\Phi_{1}(\zeta, \tau)_{i s o}=\operatorname{erfc}\left[\frac{\zeta \sqrt{b_{1}}}{2 \sqrt{\tau}}\right],
$$

where

inverting the Laplace transform, equation (22) for ramped wall concentration becomes

$$
\Phi_{1}(\zeta, \tau-1)=\left[\left(\operatorname{erfc}\left(\frac{\zeta}{2} \sqrt{\frac{b_{1}}{\tau}}\right)\right)\left(\tau+\frac{b_{1} \zeta^{2}}{2}\right)-\zeta \sqrt{b_{1}} \sqrt{\frac{\tau}{\pi} e^{-b_{1} \zeta / 4 \tau}}\right]
$$

3.3. Solution of Momentum Equation. We can get the following result by applying the Laplace transform technique to equation (10) and using equation (13): 


$$
\begin{aligned}
\bar{w}(\zeta, p)= & -\frac{1}{p}\left[\frac{m_{2}(1+\lambda p)(1-\exp (-p))}{p^{2}[\psi \lambda p+\psi-b]}\right] \exp (-\zeta \sqrt{\psi p(1+\lambda p)}) \\
& -\frac{1}{p}\left[\frac{m_{3}(1+\lambda p)(1-\exp (-p))}{p^{2}\left[\psi \lambda p+\psi-b_{1}\right]}\right] \exp (-\zeta \sqrt{\psi p(1+\lambda p)}) \\
& +\frac{1}{p}\left[\frac{m_{2}(1+\lambda p)(1-\exp (-p))}{p^{2}[\psi \lambda p+\psi-b]}\right] \exp (-\zeta \sqrt{b} \sqrt{p}) \\
& +\frac{1}{p}\left[\frac{m_{3}(1+\lambda p)(1-\exp (-p))}{p^{2}\left[\psi \lambda p+\psi-b_{1}\right]}\right] \exp \left(-\xi \sqrt{b_{1}} \sqrt{p}\right) .
\end{aligned}
$$

Equation (26) can be written in more simplified form as

$$
\bar{w}(\zeta, p)=\frac{1-\exp (-p)}{p}\left[\bar{\Psi}(\zeta, p)+\bar{\Psi}_{1}(\zeta, p)+\bar{\Psi}_{2}(\zeta, p)+\bar{\Psi}_{3}(\zeta, p)\right]
$$

where $\bar{\Psi}(\zeta, p), \bar{\Psi}_{1}(\zeta, p), \bar{\Psi}_{2}(\zeta, p)$, and $\bar{\Psi}_{3}(\zeta, p)$ are given as

$$
\begin{gathered}
\bar{\Psi}(\zeta, p)=-\left[\frac{m_{2}(1+\lambda p)}{p^{2}[\psi \lambda p+\psi-b]}\right] \exp (-\zeta \sqrt{\psi p(1+\lambda p)}), \\
\bar{\Psi}_{1}(\zeta, p)=-\left[\frac{m_{3}(1+\lambda p)}{p^{2}\left[\psi \lambda p+\psi-b_{1}\right]}\right] \exp (-\zeta \sqrt{\psi p(1+\lambda p)}), \\
\bar{\Psi}_{2}(\zeta, p)=\left[\frac{m_{2}(1+\lambda p)}{p^{2}[\psi \lambda p+\psi-b]}\right] \exp (-\zeta \sqrt{b} \sqrt{p}), \\
\bar{\Psi}_{3}(\zeta, p)=\left[\frac{m_{3}(1+\lambda p)}{p^{2}\left[\psi \lambda p+\psi-b_{1}\right]}\right] \exp \left(-\zeta \sqrt{b_{1}} \sqrt{p}\right) .
\end{gathered}
$$

By taking the invers Laplace transform equations (27)-(31), we get the following form:

$$
\bar{w}(\zeta, \tau)=\int_{0}^{\tau}\left[\tau H(\tau)-(\tau-1) H(\tau-1) *\left(\Psi(\zeta, \tau-1)+\Psi_{1}(\zeta, \tau-1)+\Psi_{2}(\zeta, \tau-1)+\Psi_{3}(\zeta, \tau-1)\right)\right] \mathrm{d} \tau
$$

where $(*)$ is the convolution product: 


$$
\begin{aligned}
& \Psi(\zeta, \tau)=-\frac{m_{2}}{\psi \lambda} \int_{0}^{\tau}\left[H(\tau-\xi) \frac{\varphi \lambda-1}{\varphi^{2}}+\frac{(\tau-\xi)}{\varphi}+\exp (-\varphi(\tau-\xi)) \frac{1-\varphi \lambda}{\varphi^{2}}\right] f_{1}(\zeta, \tau-\xi) \mathrm{d} \tau \\
& \Psi_{1}(\zeta, \tau)=-\frac{m_{3}}{\psi \lambda} \int_{0}^{\tau}\left[H(\tau-\xi) \frac{\varphi \lambda-1}{\varphi^{2}}+\frac{(\tau-\xi)}{\varphi}+\exp (-\varphi(\tau-\xi)) \frac{1-\varphi \lambda}{\varphi^{2}}\right] f_{1}(\zeta, \tau-\xi) \mathrm{d} \tau \\
& f_{1}(\zeta, \tau-\xi)=\left[\left(\operatorname{erfc}\left(\frac{\xi \psi}{2} \sqrt{\frac{\lambda \psi}{\tau}}\right)\right)\left(\tau+\frac{\psi \lambda^{2}}{2}\right)-\zeta \sqrt{\psi \lambda} \sqrt{\frac{\tau}{\psi \lambda} e^{-\psi \lambda \zeta / 4 \tau}}\right], \\
& \Psi_{2}(\zeta, \tau)=\frac{m_{2}}{\psi \lambda} \int_{0}^{\tau}\left[H(\tau-\xi) \frac{\varphi \lambda-1}{\varphi^{2}}+(\tau-\xi) \frac{1}{\varphi}+\exp (-\varphi(\tau-\xi)) \frac{1-\varphi \lambda]}{\varphi^{2}}\right] \frac{\zeta \sqrt{b} \exp \left(-\zeta^{2} b / 4 \tau\right)}{2 \tau \sqrt{\pi \tau}} \mathrm{d} \tau, \\
& \Psi_{3}(\zeta, \tau)=\frac{m_{3}}{\psi \lambda} \int_{0}^{\tau} H(\tau-\xi) \frac{\varphi \lambda-1}{\varphi^{2}}+\frac{(\tau-\xi)}{\varphi}+\exp (-\varphi(\tau-\xi)) \frac{1-\varphi \lambda}{\varphi^{2}} \frac{\zeta \sqrt{b_{1}} \exp \left(-\zeta^{2} b_{1} / 4 \tau\right)}{2 \tau \sqrt{\pi \tau}} \mathrm{d} \tau \text {. }
\end{aligned}
$$

\section{Limiting Cases}

For validation of the present work, some limiting cases are described as follows.
Case 1. In the absence of $\mathrm{Gm} \longrightarrow 0$, our solutions were reduced to the following form:

$$
\begin{aligned}
& \bar{\Theta}(\zeta, p)=\frac{1}{p^{2}} e^{-\zeta \sqrt{b p}}-\frac{e^{-p}}{p^{2}} e^{-\zeta \sqrt{b p}} \\
& \bar{w}(\zeta, p)=-\left[\frac{m_{2}(1+\lambda p)(1-\exp (-p))}{p^{2}[\psi \lambda p+\psi-b]}\right] \exp (-\zeta \sqrt{\psi p(1+\lambda p)})+\left[\frac{m_{3}(1+\lambda p)(1-\exp (-p))}{p^{2}[\psi \lambda p+\psi-b]}\right] \exp (-\zeta \sqrt{b p})
\end{aligned}
$$

By the inverse Laplace transform technique applied using the convolution theorem, we obtain the following results which are relatively same to results Arif et al. [4].

$$
\Theta_{i s o}(\zeta, \tau)=\operatorname{erfc}\left[\frac{\zeta \sqrt{b}}{2 \sqrt{\tau}}\right],
$$

$$
\Theta(\zeta, \tau)_{\mathrm{ramp}}=\Theta_{1}(\zeta, \tau)-\Theta_{1}(\zeta, \tau-1) H(\tau-\xi) .
$$

Solution of isothermal temperature is

$$
\begin{aligned}
& \Theta_{1}(\zeta, \tau)=\left[\left(\operatorname{erfc}\left(\frac{\zeta}{2} \sqrt{\frac{b}{\tau}}\right)\right)\left(\tau+\frac{b \zeta^{2}}{2}\right)-\zeta \sqrt{b} \sqrt{\frac{\tau}{\pi} e^{-(b \zeta / 4 \tau)}}\right], \\
& \Psi(\zeta, \tau)=-\frac{m_{2}}{\psi \lambda} \int_{0}^{t}\left[H(\tau-\xi) \frac{\varphi \lambda-1}{\varphi^{2}}+\frac{1}{\varphi}(\tau-\xi)+\exp (-\varphi(\tau-\xi)) \frac{1-\varphi \lambda}{\varphi^{2}}\right] f_{1}(\zeta, \tau-\xi) \mathrm{d} t, \\
& \Psi_{2}(\zeta, \tau)=-\frac{m_{3}}{\psi \lambda} \int_{0}^{\tau}\left[H(\tau-\xi) \frac{\varphi \lambda-1}{\varphi^{2}}+\frac{1}{\varphi}(\tau-\xi)+\exp (-\varphi(\tau-\xi)) \frac{1-\varphi \lambda}{\varphi^{2}}\right] \frac{\zeta \sqrt{b} \exp \left(\zeta^{2} b / 4 \tau\right)}{2 \tau \sqrt{\pi \tau}} \mathrm{d} \tau
\end{aligned}
$$

Case 2. In the absence of RWT and IWT, 


$$
\begin{aligned}
& \bar{\Theta}(\zeta, p)=\frac{1}{p} e^{-\zeta \sqrt{b} \sqrt{p}} \\
& \bar{w}(\zeta, p)=-\left[\frac{m_{2}(1+\lambda p)(1-\exp (-p))}{p^{2}[\psi \lambda p+\psi-b]}\right] \exp (\zeta \sqrt{\psi p(1+\lambda p)})+\left[\frac{m_{3}(1+\lambda p)(1-\exp (-p))}{p^{2}[\psi \lambda p+\psi-b]}\right] \exp (\zeta \sqrt{b p}) .
\end{aligned}
$$

By the inverse Laplace transform technique applied using the convolution theorem, we obtained the following results which are relatively same to results Sidra et al. [3].

$$
\begin{aligned}
& \Theta(\zeta, \tau)=\operatorname{erfc}\left[\frac{\zeta \sqrt{b}}{2 \sqrt{\tau}}\right] \\
& \Psi(\zeta, \tau)=-\frac{m_{2}}{\psi \lambda} \int_{0}^{\tau}\left[H(\tau-\xi) \frac{\varphi \lambda-1}{\varphi^{2}}+(\tau-\xi) \frac{1}{\varphi}+\exp (-\varphi(\tau-\xi)) \frac{1-\varphi \lambda}{\varphi^{2}}\right] f_{1}(\zeta, \tau-\xi) \mathrm{d} \tau, \\
& \Psi_{2}(\zeta, \tau)=-\frac{m_{3}}{\psi \lambda} \int_{0}^{\tau}\left[H(\tau-\xi) \frac{\varphi \lambda-1}{\varphi^{2}}+\frac{1}{\varphi}(\tau-\xi)+\exp (-\varphi(\tau-\xi)) \frac{1-\varphi \lambda}{\varphi^{2}}\right] \frac{\zeta \sqrt{b} \exp \left(\zeta^{2} b / 4 \tau\right)}{2 \tau \sqrt{\pi \tau}} \mathrm{d} \tau
\end{aligned}
$$

Case 3. In the absence of $\mathrm{Gr} \longrightarrow 0$, and $\lambda \longrightarrow 0$, our obtained exact results were reduced to the following form [25].

$$
\begin{aligned}
\bar{\Theta}(\zeta, p)= & \frac{1}{p^{2}} e^{-\zeta \sqrt{b p}}-\frac{e^{-p}}{p^{2}} e^{-\zeta \sqrt{b p}}, \\
\Psi(\zeta, \tau)= & -\frac{m_{2}}{\psi-b} \int_{0}^{\tau}\left[\left(\tau+\frac{\psi \zeta^{2}}{2}\right) \operatorname{erfc}\left(\frac{\zeta}{2} \sqrt{\frac{\psi}{\tau}}\right)-\zeta \sqrt{\frac{\psi \tau}{\pi}} e^{(-\zeta \psi / 4 \tau)}\right] \mathrm{d} \tau+ \\
& \frac{m_{3}}{\psi-b} \int_{0}^{\tau}\left[\left(\tau+\frac{\psi \zeta^{2}}{2}\right) \operatorname{erfc}\left(\frac{\zeta}{2} \sqrt{\frac{b}{\tau}}\right)-\zeta \sqrt{\frac{b \tau}{\pi}} e^{-\zeta^{2} \psi}\right] \mathrm{d} \tau .
\end{aligned}
$$

\section{Nusselt Number, Sherwood Number, and Skin Friction}

5.1. Nusselt Number. The dimensional form of Nusselt number for Maxwell nanofluid is given by

$$
\mathrm{Nu}=-\left.\frac{k_{n f}}{k_{f}}\left(\frac{\sqrt{v \tau}}{T_{1 w}-T_{1 \infty 0}}\right) \frac{\partial T_{1}}{\partial y_{1}}\right|_{y=0} .
$$

By using equations (6) and (7), the dimensionless form of equation (40) becomes

$$
\mathrm{Nu}=-\left.\frac{k_{n f}}{k_{f}} \frac{\partial \Theta}{\partial \zeta}\right|_{\zeta=0} .
$$

5.2. Sherwood Number. The dimensional form of Sherwood number for the Maxwell nanofluid is given by

$$
S_{h}=-\left.D_{n f}\left(\frac{\sqrt{\nu \tau}}{C_{1 w}-C_{1 \infty}}\right) \frac{\partial C_{1}}{\partial y_{1}}\right|_{y_{1}=0} .
$$

By using equations (6) and (7), the dimensionless form of equation (42) becomes

$$
S_{h}=-\left.D_{n f} \frac{\partial \Phi}{\partial \zeta}\right|_{\zeta=0} .
$$

5.3. Skin Friction. The dimensional form of nonzero shear stress for Maxwell fluid is given as

$$
\tau_{x y}^{*}=\left.\frac{\mu_{f}}{\left(1+\lambda_{1}\left(\partial / \partial t_{1}\right)\right)} \frac{\partial u_{1}}{\partial y_{1}}\right|_{y_{1}=0} .
$$

For Maxwell nanofluid, equation (46) takes the following form: 


$$
\tau_{x y}^{*}=\left.\frac{\mu_{n f}}{\left(1+\lambda_{1}\left(\partial / \partial t_{1}\right)\right)} \frac{\partial u_{1}}{\partial y_{1}}\right|_{y_{1}=0} .
$$

By using equations (6) and (7), the dimensionless form of equation (47) becomes

$$
\tau_{x y}=\left.\frac{\mu_{f}}{(1-\phi)^{2.5}} \frac{1}{(1+\lambda(\partial / \partial \tau))} \frac{\partial w}{\partial \zeta}\right|_{\zeta=0},
$$

where $\tau_{x y}=\tau_{x y}^{*} \sqrt{\nu\left(t_{1} / \tau_{0}\right)} / \mu_{f} U_{0}$ which is the dimensionless form of nonzero is shear stress and $\lambda=\lambda_{1} / \tau_{0}$ is the dimensionless Maxwell parameter.

The mathematical representation for the skin friction $\left(S_{f}\right)$ for Maxwell nanofluid is given as

$$
S_{f}=\left.\frac{1}{(1-\phi)^{2.5}} \frac{1}{(1+\lambda(\partial / \partial \tau))} \frac{\partial w}{\partial \zeta}\right|_{\zeta=0} .
$$

\section{Results and Discussion}

We studied the exact solutions of Maxwell fluid flow with ramped and isothermal wall boundary conditions in this study. We used EO as the base fluid and widely scattered $\mathrm{MoS}_{2}$ particles to improve the thermal conductivity of EO. Through the graphical analysis, the effect of $\lambda, \phi, \mathrm{Gm}$, and Sc on the velocity profile $w(\zeta, t)$, temperature distribution $\Theta(\zeta, t)$, and concentration distribution $\Phi(\zeta, t)$ are shown. More specially, Figure 1 is the physical configuration of the problem. In Figures $2-7$, the impact of various parameters on the velocity profile has been seen. The effect of sundry parameters on temperature is shown graphically in Figures 8 and 9. Finally, the impact of embedded parameters on concentration distribution is shown graphically in Figures 10-13.

The influence of various values of time $\tau$ on velocity $w(\zeta, t)$ is sketched in Figure 2. The figure illustrates that the magnitude of velocity distribution increases when RWT, IWT, RWC, and IWC increase. The impact of different values of the Maxwell parameter $\lambda$ on the velocity profile is demonstrated in Figure 3. From the figure, it is clear that the Maxwell fluid velocity increases with relaxation time $\lambda$. It is because, when we raise the value of $\lambda$, the gradual response obtains to shear stress and accelerates the Maxwell nanofluid velocity. Figures 4 and 5 indicate the impact of sundry values of $\mathrm{Gm}$ and $\mathrm{Gr}$ on velocity distribution, respectively. It is seen that $\mathrm{Gm}$ and $\mathrm{Gr}$ accelerate the fluid motion. The fact behind this is that increasing $\mathrm{Gm}$ and $\mathrm{Gr}$ emerges to improve the thermal and mass buoyancy forces that raise the fluid motion. Increasing the mass buoyancy forces, the viscosity of the Maxwell nanofluid decreases which accelerates the fluid motion. Figure 6 shows how Sc influences the Maxwell nanofluid velocity. The Maxwell nanofluid velocity is found to be reduced when Sc is increased. Since Sc is the ratio of mass diffusion to viscous forces, increasing Sc increases viscous forces, while decreasing mass diffusion, decreasing velocity. Figure 7 shows the influence of various values of $\phi$ on the Maxwell nanofluid velocity. By increasing the volume fraction, the Maxwell nanofluid velocity retards for both cases RWT, IWT, RWC, and IWC. The physics behind this is that by increasing $\phi$, the flow becomes more vicious results the friction forces that retard the velocity of nanofluid.

Figure 8 explains the impact of time on temperature distribution. By increasing $\tau$, RWT rises, while IWT remains. Figure 9 illustrates the behavior of $\phi$ on temperature profile. From the figure, one can clearly see that increasing $\phi$ increases the temperature profile. The physics behind this behavior of rising temperature is the collision between the molecules of Maxwell nanofluid rises, and as a result, the temperature rises.

Figure 10 shows how is the effect of $\tau$ on the concentration profile. We can see from the graph that increasing $\tau$ increases RWC while keeping IWC constant. Figures 11 and 12 show the effects of $\phi$ and Sc on the distribution of concentrations, respectively. The concentration distribution decreases for significant values of $\phi$ and Sc, as can be seen in both of these figures. The explanation for this behavior is that as the concentration distribution slows down, viscous forces rise. Sc, on either extreme, is the ratio of mass diffusion to viscous forces. As Sc rises, viscous forces increase when mass diffusion decreases, resulting in a decreasing of the concentration distribution.

Figure 13 illustrates the difference between our results and those of the published studies of Sidra et al. [3]. Our findings strongly overlap with the reported results, as seen in the figure, when RWT and IWT are not available. From Figure 14, our findings are consistent with the findings of the published article of Arif et al. [4] by taking Gm $\longrightarrow 0$. Figure 15 compares our findings to the findings of the published article of Khalid et al. [25] to validate our obtained solutions. From this figure, our results matched the results of Khalid et al. [25] by taking $\lambda \longrightarrow 0$ and $\mathrm{Gm} \longrightarrow 0$.

Table 2 presents how various embedded parameters affect the skin friction of a Maxwell nanofluid flow. For both ramped and isothermal wall temperatures and concentrations, skin friction is determined. Skin friction for isothermal wall conditions is higher than skin friction for ramped wall conditions. The rate of heat transfer for ramped and isothermal wall temperatures is given in Tables 3 and 4 . When considering ramped wall temperature, the heat transfer rate increases by $12.895 \%$, and when considering isothermal wall temperature, the heat transfer rate increases by $12.899 \%$. Sherwood numbers for ramped and isothermal wall concentrations are given in Tables 5 and 6 . We found that $3.030 \%$ of mass transfer decreases for ramped wall concentration and $3.104 \%$ increases by considering isothermal wall concentration for $\phi=0.04$. 


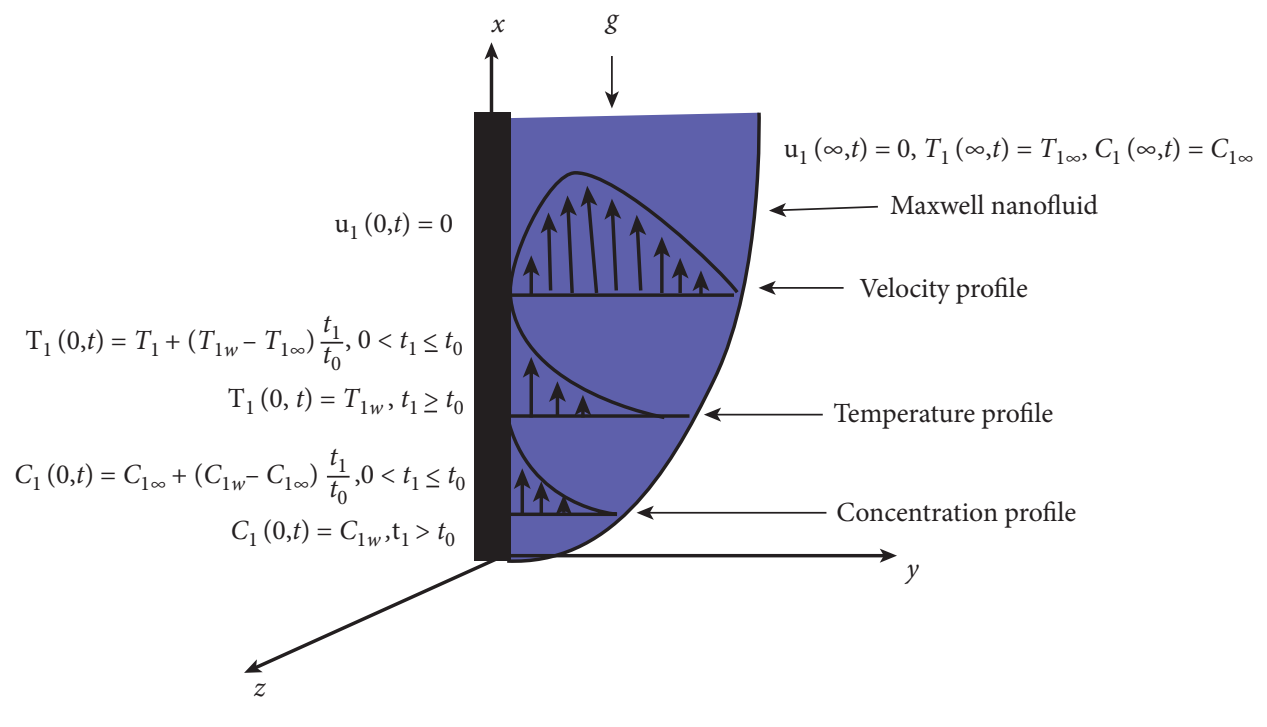

Figure 1: Physical configuration of the problem.

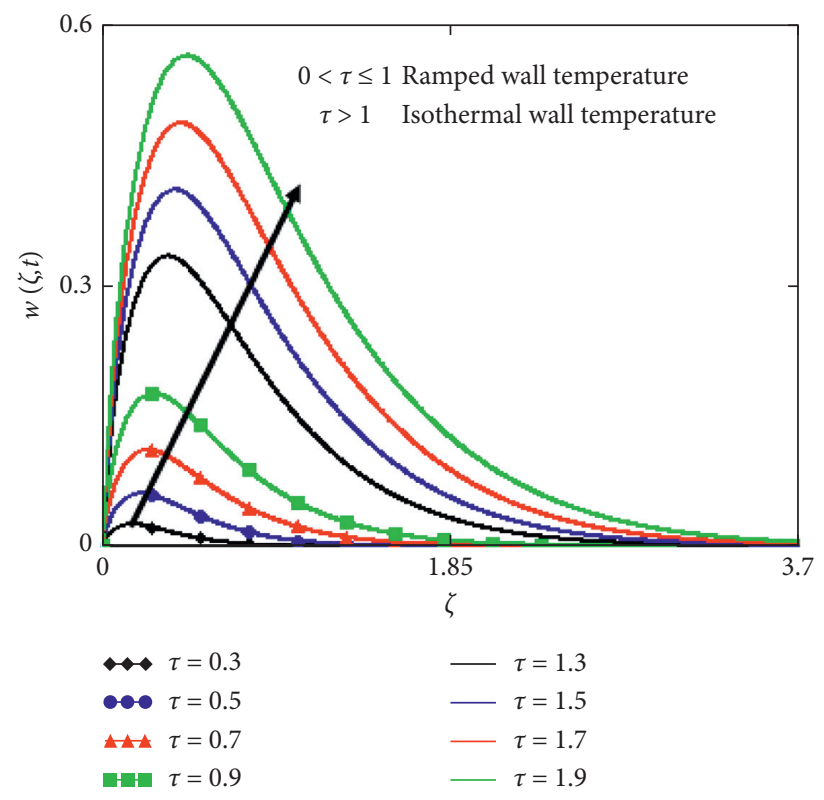

Figure 2: The effect of different values of $\tau$ on velocity distribution of $\mathrm{MoS}_{2}$ EO-based Maxwell nanofluid when $\phi=0.02, \mathrm{Gr}=0.05, \operatorname{Pr}=6300, \mathrm{sc}=15, \mathrm{Gm}=0.5$. 


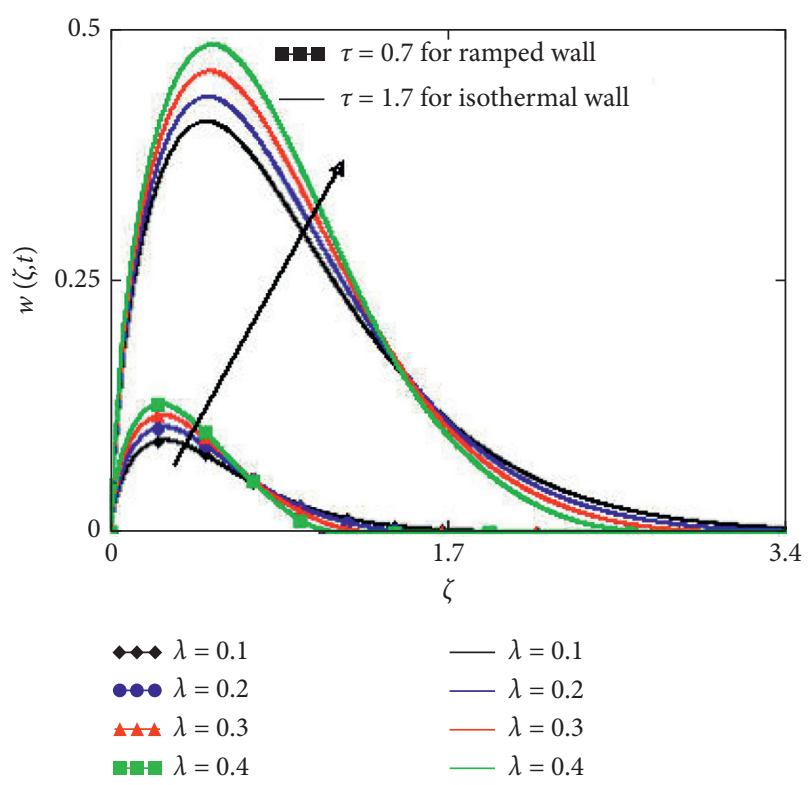

Figure 3: The impact of sundry values of $\lambda$ on the velocity distribution of Maxwell nanofluid when $\phi=0.01, \mathrm{Gr}=0.05, \mathrm{Pr}=$ $6300, \mathrm{sc}=9, \tau=0.7, \tau=1.7, \mathrm{Gm}=0.5$.

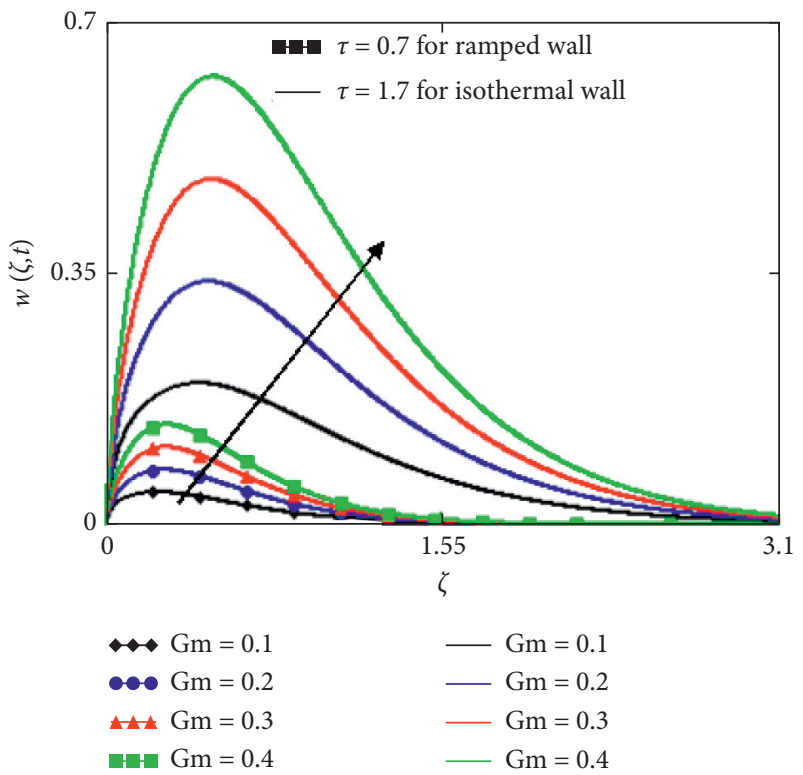

FIgURE 4: The impact of different values of $\mathrm{Gm}$ on velocity distribution of $\mathrm{MoS}_{2}$ EO-based Maxwell nanofluid when $\phi=0.02, \mathrm{Gr}=0.05, \operatorname{Pr}=6300, \mathrm{sc}=9, \tau=0.7, \tau=1.7, \lambda=0.1$.

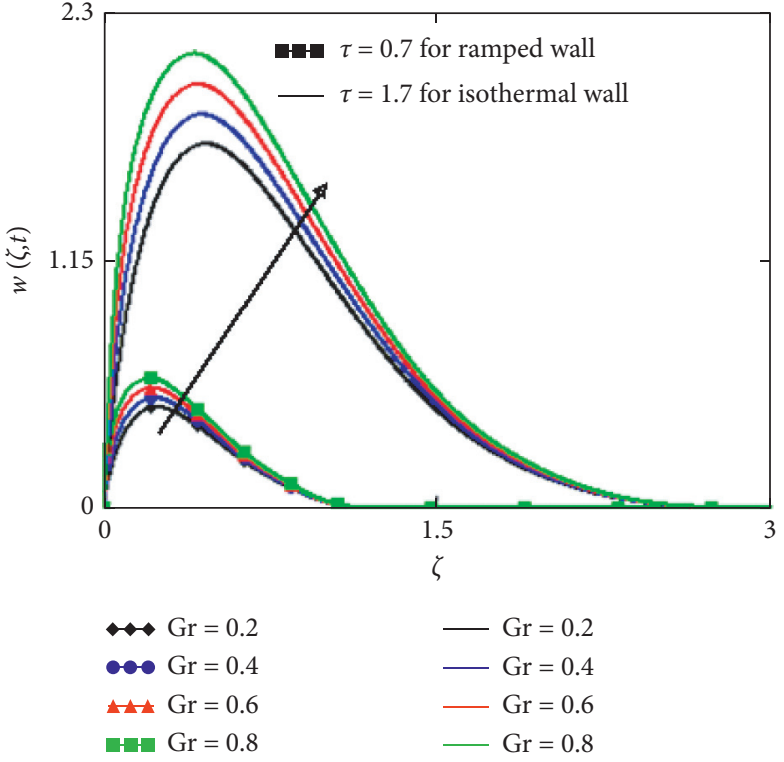

Figure 5: The impact of different values of Gr on velocity distribution of $\mathrm{MoS}_{2}$ EO-based Maxwell nanofluid when $\phi=0.01, \mathrm{Gm}=3, \operatorname{Pr}=6300, \mathrm{sc}=15, \tau=0.7, \tau=1.7, \lambda=0.4$.

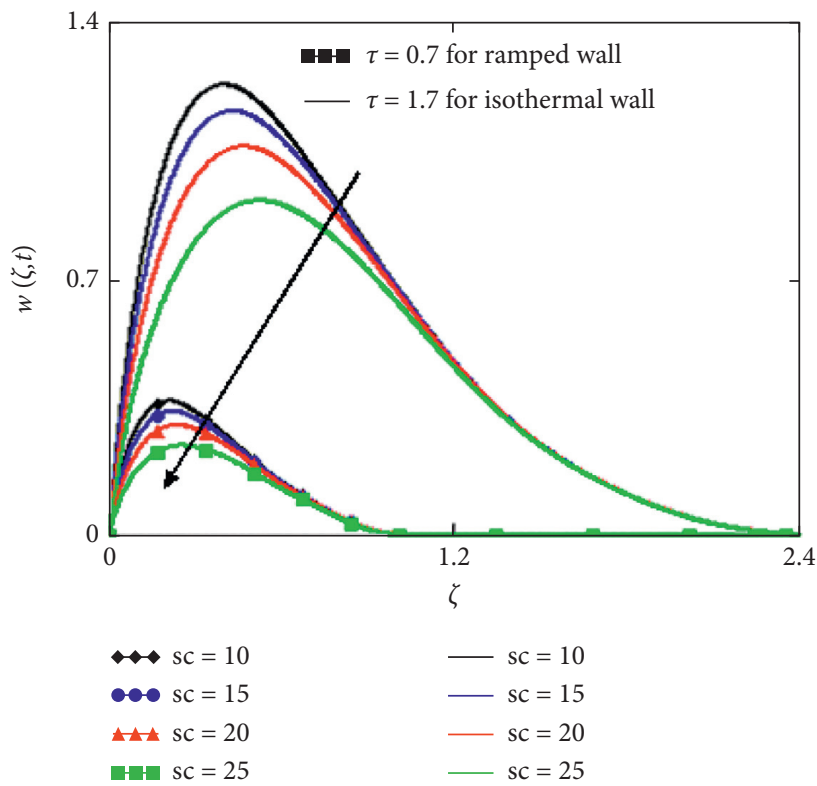

FIgURE 6: The impact of different values of Sc on velocity distribution of $\mathrm{MoS}_{2}$ EO-based Maxwell nanofluid when $\phi=0.02, \mathrm{Gr}=0.05, \mathrm{Gm}=0.9, \tau=0.7, \tau=1.7, \lambda=0.5, \operatorname{Pr}=6300$. 


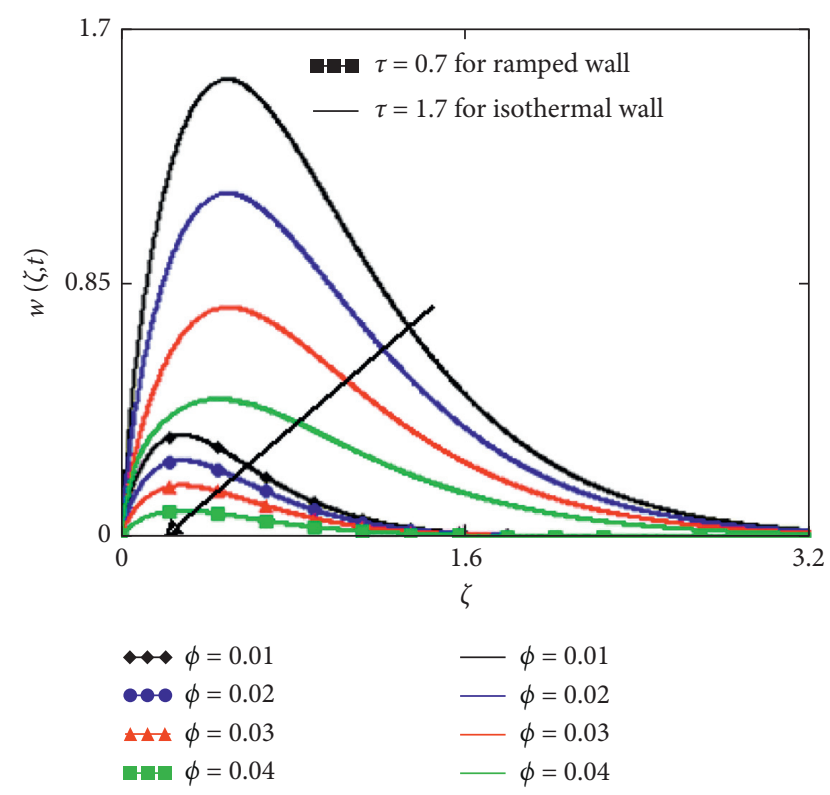

Figure 7: The impact of different values of $\phi$ on the velocity distribution of $\mathrm{MoS}_{2}$ EO-based Maxwell nanofluid when $\mathrm{Gr}=0.05, \mathrm{Pr}=6300, \mathrm{Gm}=1, \mathrm{sc}=9, \tau=0.7, \tau=1.7, \lambda=0.1$.

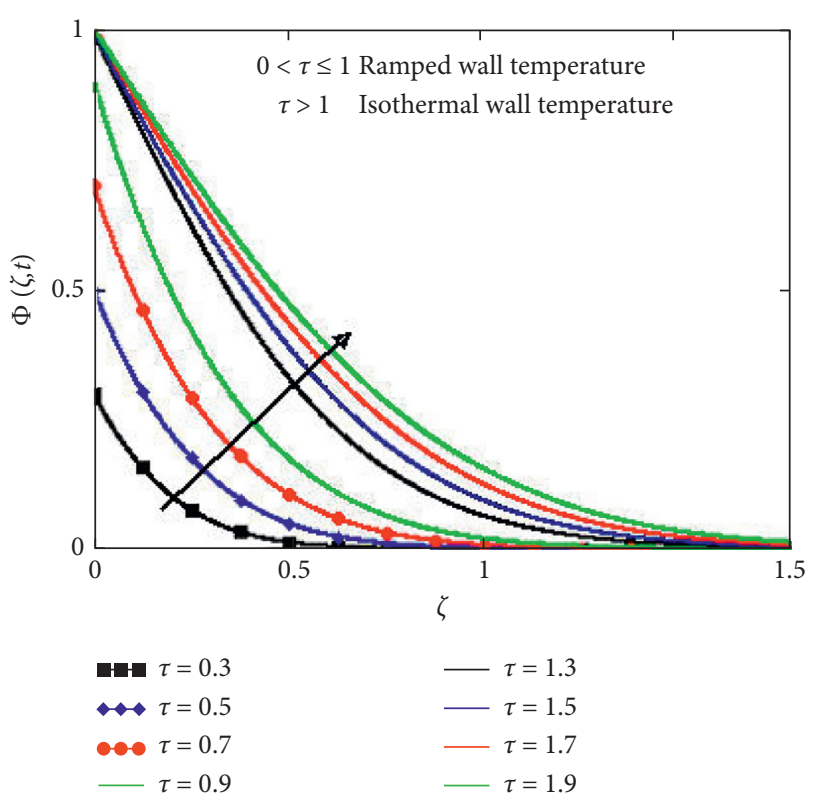

FIgURE 8: The impact of different values of $\tau$ on temperature distribution of $\mathrm{MoS}_{2}$ EO-based Maxwell nanofluid when $\phi=0.05, \operatorname{Pr}=6300$.

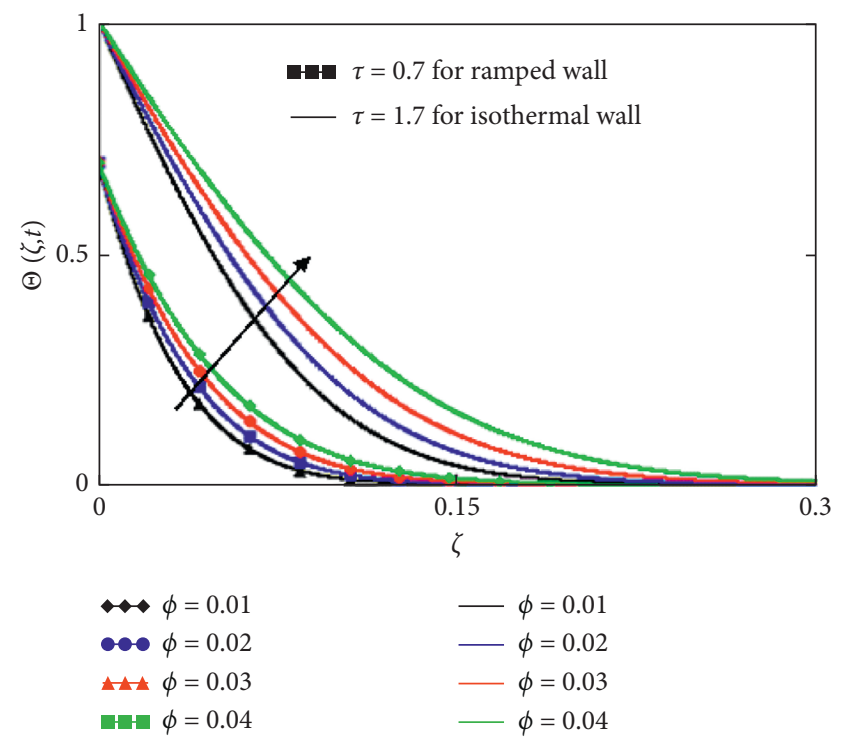

Figure 9: The impact of different values of $\phi$ on temperature distribution of $\mathrm{MoS}_{2}$ EO-based Maxwell nanofluid for when $\operatorname{Pr}=6300, \tau=0.7, \tau=1.7$.

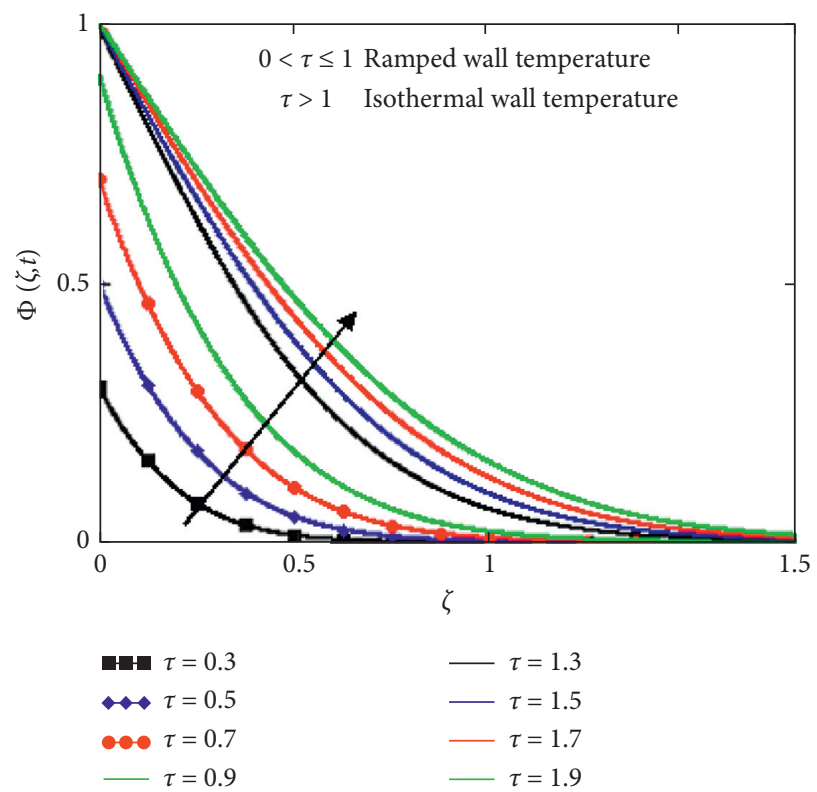

Figure 10: The effect of different value of $\tau$ on concentration distribution of $\mathrm{MoS}_{2}$ EO-based Maxwell nanofluid when $\phi=0.1, \mathrm{sc}=5$. 


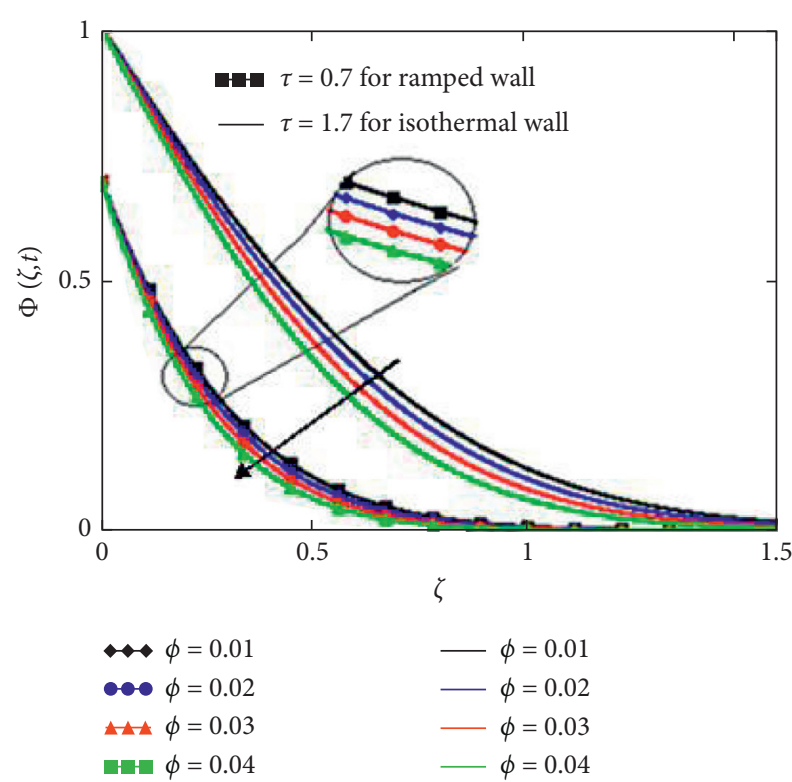

Figure 11: The impact of different values of $\phi$ on concentration distribution of $\mathrm{MoS}_{2}$ EO-based Maxwell nanofluid when $\mathrm{sc}=5, \tau=0.7, \tau=1.7$.

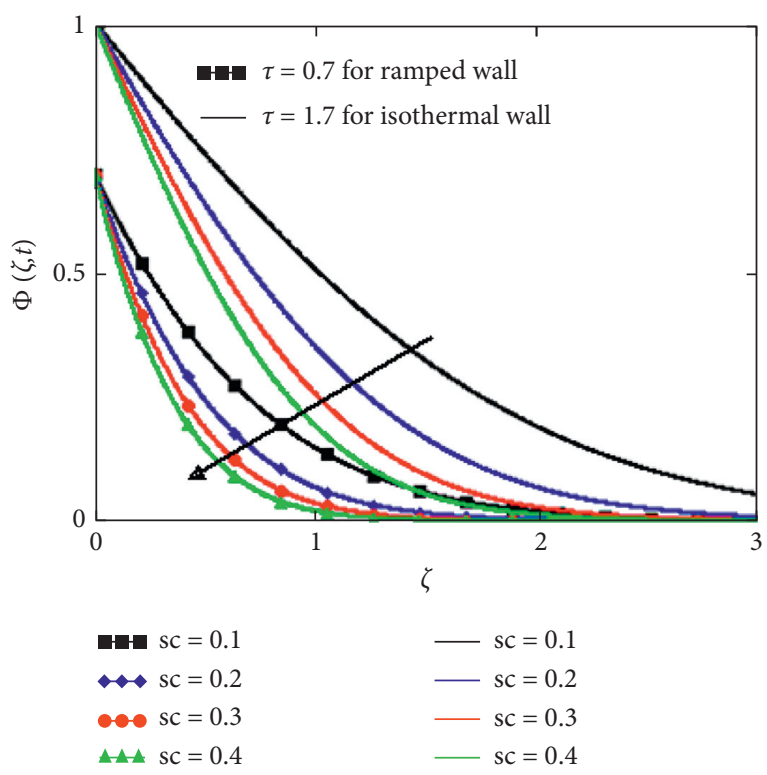

FIgURE 12: The effect of different values of Sc on concentration distribution of $\mathrm{MoS}_{2}$ EO-based Maxwell nanofluid when $\phi=0.01, \tau=0.7, \tau=1.7$.

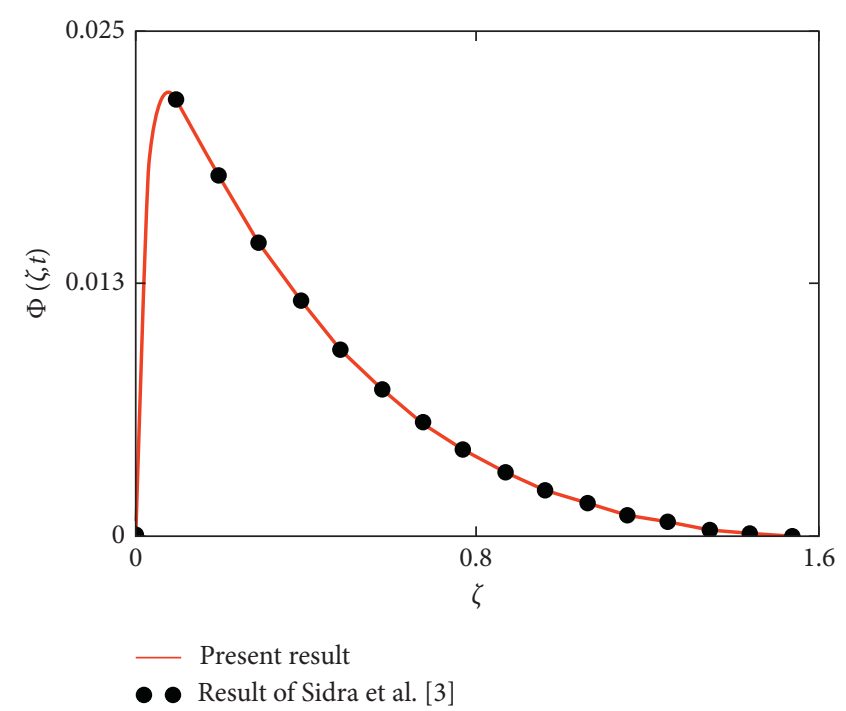

FIgURE 13: Velocity distribution of $\mathrm{MoS}_{2}$ EO-based Maxwell nanofluid when $\mathrm{Gr}=0.5 \phi=0.01, \mathrm{Gm}=0, \operatorname{Pr}=6300, \mathrm{sc}=15, \tau=1.5, \lambda=0.4$.

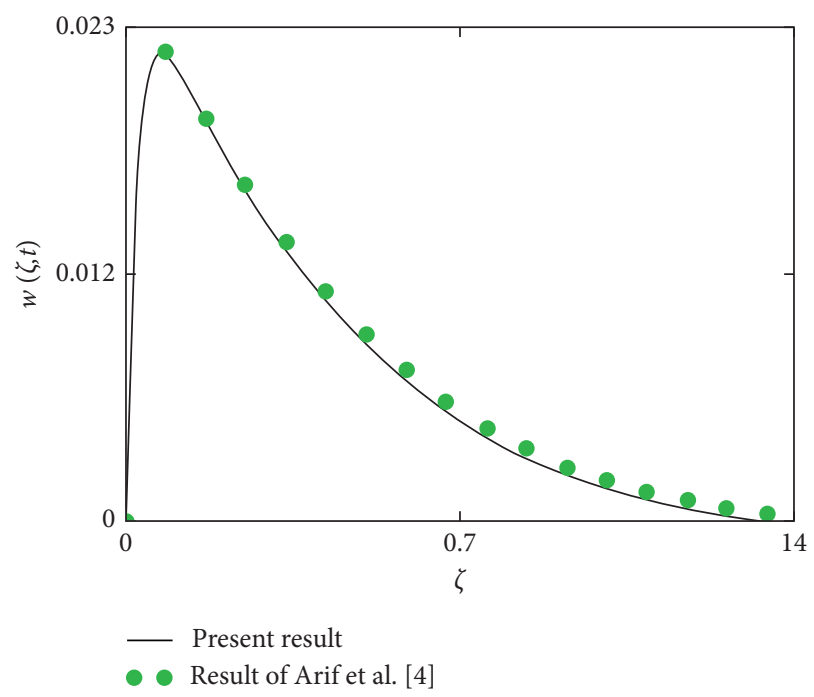

Figure 14: Velocity distribution of $\mathrm{MoS}_{2}$ EO-based Maxwell nanofluid when $\phi=0.01, \mathrm{Gm}=0, \mathrm{Gr}=5, \operatorname{Pr}=6300, \mathrm{sc}=15, \tau_{0}=$ $0.7, \tau=1.7, \lambda=0.4$. 


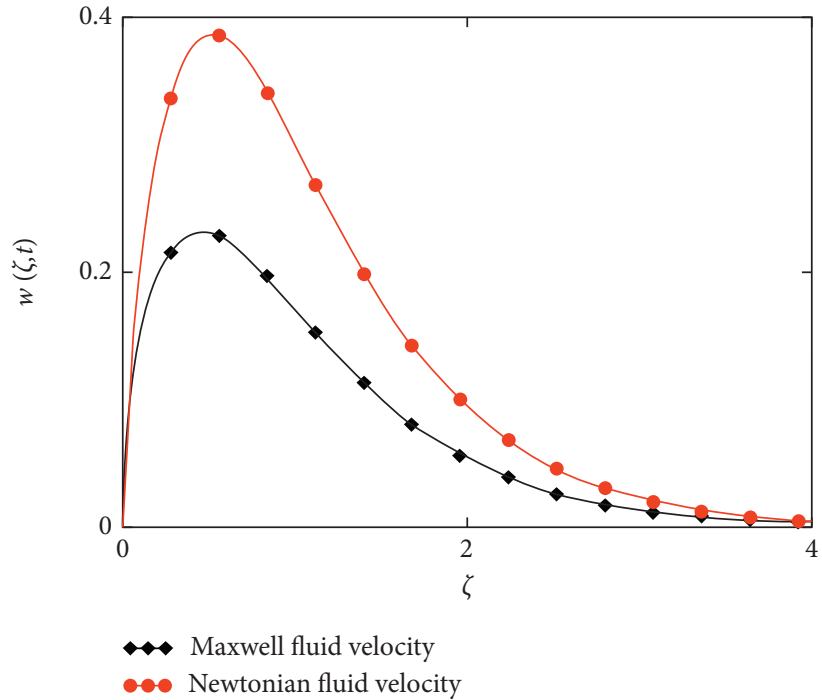

FIgure 15: Velocity distribution of $\mathrm{MoS}_{2}$ EO-based Maxwell nanofluid for the different value of $\phi=0.01, \mathrm{Gm}=0, \mathrm{Gr}=5, \operatorname{Pr}=6300, \mathrm{sc}=15$, and $\tau=1.7$.

TABLE 2: Skin friction of RWT and IWT EO-based $\mathrm{MoS}_{2}$.

\begin{tabular}{lccccccccc}
\hline$\lambda$ & $\mathrm{Gm}$ & $\mathrm{Gr}$ & $\mathrm{Pr}$ & $\tau$ & $\tau_{0}$ & $\phi$ & $\mathrm{Sc}$ & $C_{f_{\text {ramp }}}$ & $C_{f_{\text {iso }}}$ \\
\hline 0.1 & 0.5 & 0.5 & 6300 & 1.7 & 0.7 & 0.02 & 10 & \\
$\mathbf{0 . 2}$ & 0.5 & 0.5 & 6300 & 1.7 & 0.7 & 0.02 & 10 & 10.598 \\
0.1 & $\mathbf{1 . 5}$ & 0.5 & 6300 & 1.7 & 0.7 & 0.02 & 10 & 10.581 \\
0.1 & 0.5 & $\mathbf{1 . 5}$ & 6300 & 1.7 & 0.7 & 0.02 & 10 & 22.749 \\
0.1 & 0.5 & 0.5 & $\mathbf{7 3 0 0}$ & 1.7 & 0.7 & 0.02 & 10 & 25.659 \\
0.1 & 0.5 & 0.5 & 6300 & $\mathbf{1 . 9}$ & 0.7 & 0.02 & 10 & 9.361 \\
0.1 & 0.5 & 0.5 & 6300 & 1.7 & $\mathbf{1 . 9}$ & 0.02 & 10 & 12.764 \\
0.1 & 0.5 & 0.5 & 6300 & 1.7 & 0.7 & $\mathbf{0 . 0 3}$ & 10 & 13.43 \\
0.1 & 0.5 & 0.5 & 6300 & 1.7 & 0.7 & 0.02 & $\mathbf{1 5}$ & 9.323 \\
\hline
\end{tabular}

The values in bold show the variation in parameter values against skin friction.

TABle 3: Distinction in the rate of heat transfer RWT EO-based $\mathrm{MoS}_{2}$.

\begin{tabular}{lccccc}
\hline$\phi$ & $\tau$ & $\tau_{0}$ & $\operatorname{Pr}$ & $\mathrm{Nu}_{\text {ramp }}$ & Heat transfer enhancement $(\%)$ \\
\hline 0.00 & 1.7 & 0.7 & 6300 & 23.132 & 4.750 \\
0.01 & 1.7 & 0.7 & 6300 & 24.231 & 7.971 \\
0.02 & 1.7 & 0.7 & 6300 & 24.976 & 9.597 \\
0.03 & 1.7 & 0.7 & 6300 & 25.352 & 12.895 \\
0.04 & 1.7 & 0.7 & 6300 & 26.115 & \\
\hline
\end{tabular}

TABLE 4: Variation in the rate of heat transfer IWT EO-based $\mathrm{MoS}_{2}$.

\begin{tabular}{lccccc}
\hline$\phi$ & $\tau$ & $\tau_{0}$ & $\operatorname{Pr}$ & $\mathrm{Nu}_{i s o}$ & Heat transfer enhancement $(\%)$ \\
\hline 0.00 & 1.7 & 0.7 & 6300 & 13.404 & 5.460 \\
0.01 & 1.7 & 0.7 & 6300 & 14.404 & 7.967 \\
0.02 & 1.7 & 0.7 & 6300 & 14.472 & 8.109 \\
0.03 & 1.7 & 0.7 & 6300 & 14.491 & 12.899 \\
0.04 & 1.7 & 0.7 & 6300 & 15.133 & \\
\hline
\end{tabular}


TABLE 5: Variation in the rate of mass transfer RWC EO-based $\mathrm{MoS}_{2}$.

\begin{tabular}{lccccc}
\hline$\phi$ & $\tau$ & $\tau_{0}$ & $S c$ & $S_{h_{\text {ramp }}}$ & Decrease in mass distribution (\%) \\
\hline 0.00 & 1.7 & 0.7 & 5 & 1.056 & 1.04 \\
0.01 & 1.7 & 0.7 & 5 & 1.034 & 2.083 \\
0.02 & 1.7 & 0.7 & 5 & 1.029 & 2.556 \\
0.03 & 1.7 & 0.7 & 5 & 1.024 & 3.030 \\
0.04 & 1.7 & 0.7 & 5 & \\
\hline
\end{tabular}

TABLE 6: Variation in the rate of mass transfer IWC EO-based $\mathrm{MoS}_{2}$.

\begin{tabular}{lccccc}
\hline$\phi$ & $\tau$ & $\tau_{0}$ & Sc & $S_{h_{i s o}}$ & Decrease in mass distribution (\%) \\
\hline 0.00 & 1.7 & 0.7 & 5 & 0.612 & 1.470 \\
0.01 & 1.7 & 0.7 & 5 & 0.603 & 2.124 \\
0.02 & 1.7 & 0.7 & 5 & 0.599 & 2.614 \\
0.03 & 1.7 & 0.7 & 5 & 0.596 & 3.104 \\
0.04 & 1.7 & 0.7 & 5 & 0.593 & \\
\hline
\end{tabular}

\section{Concluding Remarks}

The aim of this study is to find exact solutions for mass and heat transfer in a Maxwell nanofluid flow with ramped and isothermal wall temperatures and concentration boundary conditions. The Laplace transform method is used to obtain precise solutions for velocity, temperature, and concentration distributions. Additionally, $\mathrm{MoS}_{2}$ nanoparticles are dispersed in EO. Additionally, the results obtained for ramped and isothermal wall temperature and concentration are analyzed numerically using various graphs and tables.

The significant outcomes of our study are as follows:

(i) The velocity of Maxwell nanofluid slows down by increasing the amount of $\mathrm{MoS}_{2}$ nanoparticles

(ii) The velocity of a Newtonian fluid is greater than the velocity of a Maxwell nanofluid

(iii) RWT and RWC have narrower velocity, temperature, and concentration distributions, while IWT and IWC have greater distributions.

(iv) By rising $\lambda, \mathrm{Gm}, \mathrm{Gr}$, and $\tau$, the Maxwell nanofluid velocity increases

(v) By rising the value of $\phi$ for RWT enhanced heat transfer up to $12.895 \%$ while $12.899 \%$ for IWT of regular EO

(vi) The rate of mass transfer for RWC decreases to $3.030 \%$ while $3.104 \%$ for IWC of regular EO

(vii) Using nanoparticles in the engine oil increases the heat transfer rate which will off course increase life and efficiency of engines.

(viii) $\mathrm{MoS}_{2}$ is also used as dry lubricant. By making use of $\mathrm{MoS}_{2}$ in the engine oil, it increases the lubricity of EO which will help you to decrease the corrosion and friction of the engine parts.
Abbreviations
$U_{0}: \quad$ Uniform velocity
$u_{1}$ : Dimensional velocity in $x$-direction

$w: \quad$ Dimensionless velocity in $x$-direction

$T_{1}$ : $\quad$ Dimensional temperature in $x$-direction

$C_{1}$ : Dimensional concentration in $x$-direction

$T_{1 \infty}$ : Ambient temperature

$C_{100}$ : Constant concentration

$T_{1 w}$ : Wall temperature

$C_{1 w}$ : Wall concentration

$H(\tau)$ : Heaviside step function

$k_{f}: \quad$ Thermal conductivity of base fluid

$k_{n f}: \quad$ Thermal conductivity of nanofluid

$k_{s}: \quad$ Thermal conductivity of nanoparticles

$g_{x}: \quad$ Gravitational acceleration

Sf: $\quad$ Skin friction

Nu: $\quad$ Nusselt number

$S_{h}: \quad$ Sherwood number

Pr: $\quad$ Prandtl number

Gr: $\quad$ Thermal Grashof number

Gm: $\quad$ Mass Grashof number

Sc: $\quad$ Schmidt number

$t$ : Time

$t_{0}: \quad$ Characteristic time

$\lambda_{1}: \quad$ Dimensional Maxwell parameter

$\lambda: \quad$ Dimensionless Maxwell parameter

$\Theta: \quad$ Dimensionless fluid temperature

$\Phi: \quad$ Dimensionless fluid concentration

$\tau: \quad$ Dimensional time

$\zeta: \quad$ Dimensionless space variable

$\rho_{f}: \quad$ Density of base fluid

$\rho_{n f}: \quad$ Density of nanofluid

$\rho_{s}: \quad$ Density of nanoparticles

$\left(\rho c_{p}\right)_{f}$ : Heat capacitance of base fluid

$\left(\rho c_{p}\right)_{n f}$ : Heat capacitance of nanofluid

$\left(\rho c_{p}\right)_{s}:$ Heat capacitance of nanoparticles

$\beta_{f}: \quad$ Thermal expansion coefficient of base fluid

$\beta_{s}: \quad$ Thermal expansion coefficient of nanoparticles

$\beta_{n f}$ : Thermal expansion coefficient of nanofluid

$\mu_{f}: \quad$ Dynamic viscosity of base fluid

$\mu_{n f}: \quad$ Dynamic viscosity of nanofluid

$D_{f}$ : Thermal diffusivity of base fluid

$D_{n f}: \quad$ Thermal diffusivity of nanofluid 
$\phi: \quad$ Volume fraction

$\sigma: \quad$ Standard distribution.

\section{Data Availability}

The data used to support the findings of this study are included within the article.

\section{Conflicts of Interest}

The authors declare that they have no conflicts of interest.

\section{References}

[1] D. Cioranescu, V. Girault, and K. R. Rajagopal, Mechanics and Mathematics of Fluids of the Differential Type, Vol. 35, Springer, New York, NY, USA, 2016.

[2] J. C. Maxwell, A Treatise on Electricity and Magnetism, Vol. 1, Clarendon Press, Oxford, UK, 1881.

[3] S. Aman, M. Z. Salleh, Z. Ismail, and I. Khan, "Exact solution for heat transfer free convection flow of Maxwell nanofluids with graphene nanoparticles," Journal of Physics: Conference Series, vol. 890, no. 1, pp. 1742-6596, 2017.

[4] M. Arif, F. Ali, N. A. Sheikh, I. Khan, and K. S. Nisar, "Fractional model of couple stress fluid for generalized Couette flow: a comparative analysis of atangana-baleanu and caputo-fabrizio fractional derivatives," IEEE Access, vol. 7, no. 11, pp. 88643-88655, 2019.

[5] A. Khalid, I. Khan, and S. Shafie, "Heat transfer in ferrofluid with cylindrical shape nanoparticles past a vertical plate with ramped wall temperature embedded in a porous medium," Journal of Molecular Liquids, vol. 221, pp. 1175-1183, 2016.

[6] J. Zhao, L. Zheng, X. Zhang, and F. Liu, "Convection heat and mass transfer of fractional MHD Maxwell fluid in a porous medium with Soret and Dufour effects," International Journal of Heat and Mass Transfer, vol. 103, pp. 203-210, 2016.

[7] R. R. Huilgol, "On the properties of the motion with constant stretch history occurring in the Maxwell rheometer," Transactions of the Society of Rheology, vol. 13, no. 4, pp. 513-526, 1969.

[8] M. Jamil, "Effects of slip on oscillating. fractionalized Maxwell fluid," Nonlinear Engineering, vol. 5, no. 1, pp. 25-36, 2016.

[9] T. Anwar, P. Kumam, W. Watthayu, and S. Asifa, "Influence of ramped wall temperature and ramped wall velocity on unsteady magnetohydrodynamic convective Maxwell fluid flow," Symmetry, vol. 12, no. 3, p. 392, 2020.

[10] W. Na, N. A. Shah, I. Tlili, and I. Siddique, "Maxwell fluid flow between vertical plates with damped shear and thermal flux: free convection," Chinese Journal of Physics, vol. 65, no. 3, pp. 367-376, 2020.

[11] N. Raza and M. A. Ullah, "A comparative study of heat transfer analysis of fractional Maxwell fluid by using Caputo and Caputo-Fabrizio derivatives," Canadian Journal of Physics, vol. 98, no. 1, pp. 89-101, 2020.

[12] J. Sui, L. Zheng, and X. Zhang, "Boundary layer heat and mass transfer with Cattaneo-Christov double-diffusion in upperconvected Maxwell nanofluid past a stretching sheet with slip velocity," International Journal of Thermal Sciences, vol. 104, pp. 461-468, 2016.

[13] S. Wang and W. Tan, "Stability analysis of soret-driven double-diffusive convection of Maxwell fluid in a porous medium," International Journal of Heat and Fluid Flow, vol. 32, no. 1, pp. 88-94, 2011.
[14] H. J. Steeman, C. T'Joen, M. Van Belleghem, A. Janssens, and M. De Paepe, "Evaluation of the different definitions of the convective mass transfer coefficient for water evaporation into air," International Journal of Heat and Mass Transfer, vol. 52, no. 15-16, pp. 3757-3766, 2009.

[15] Y. Men, X. Liu, and T. Zhang, "Analytical solutions of heat and mass transfer process in combined gas-water heat exchanger applied for waste heat recovery," Energy, vol. 206, Article ID 118095, 2020.

[16] M. Chakkingal, R. Voigt, C. R. Kleijn, and S. Kenjereš, "Effect of double-diffusive convection with cross gradients on heat and mass transfer in a cubical enclosure with adiabatic cylindrical obstacles," International Journal of Heat and Fluid Flow, vol. 83, Article ID 108574, 2020.

[17] W.-M. Yan, C.-Y. Lee, C.-H. Li, W.-K. Li, and S. Rashidi, "Study on heat and mass transfer of a planar membrane humidifier for PEM fuel cell," International Journal of Heat and Mass Transfer, vol. 152, Article ID 119538, 2020.

[18] F. Chu, M. Su, and G. Yang, "Heat and mass transfer characteristics of ammonia regeneration in packed column," Applied Thermal Engineering, vol. 176, Article ID 115405, 2020.

[19] S. A. Hazarika, T. Deshmukhya, D. Bhanja, and S. Nath, "A novel optimum constructal fork-shaped fin array design for simultaneous heat and mass transfer application in a spaceconstrained situation," International Journal of Thermal Sciences, vol. 150, Article ID 106225, 2020.

[20] M. I. H. Khan, Z. Welsh, Y. Gu, M. A. Karim, and B. Bhandari, "Modelling of simultaneous heat and mass transfer considering the spatial distribution of air velocity during intermittent microwave convective drying," International Journal of Heat and Mass Transfer, vol. 153, Article ID 119668, 2020.

[21] A. V. Luikov, "Heat and mass transfer in capillary-porous bodies," Advances in Heat Transfer, Elsevier, vol. 1, pp. 123-184, 1964.

[22] M. B. Riaz, A. Atangana, and N. Iftikhar, "Heat and mass transfer in Maxwell fluid in view of local and non-local differential operators," Journal of Thermal Analysis and Calorimetry, vol. 143, pp. 1-17, 2020.

[23] X. Zhu, S. Chen, S. Shen, S. Ni, X. Shi, and Q. Qiu, "Experimental study on the heat and mass transfer characteristics of air-water two-phase flow in an evaporative condenser with a horizontal elliptical tube bundle," Applied Thermal Engineering, vol. 168, Article ID 114825, 2020.

[24] P. Chandran, N. C. Sacheti, and A. K. Singh, "Natural convection near a vertical plate with ramped wall temperature," Heat and Mass Transfer, vol. 41, no. 5, pp. 459-464, 2005.

[25] A. Khalid, I. Khan, and S. Shafie, "Exact solutions for free convection flow of nanofluids with ramped wall temperature," The European Physical Journal Plus, vol. 130, no. 4, p. 57, 2015.

[26] M. Narahari, "Transient free convection flow between long vertical parallel plates with ramped wall temperature at one boundary in the presence of thermal radiation and constant mass diffusion," Meccanica, vol. 47, no. 8, pp. 1961-1976, 2012.

[27] N. Ghara, S. Das, S. L. Maji, and R. N. Jana, "Effect of radiation on MHD free convection flow past an impulsively moving vertical plate with ramped wall temperature," American Journal of Scientific and Industrial Research, vol. 3, no. 6, pp. 376-386, 2012.

[28] S. U. Haq, I. Khan, F. Ali, A. Khan, and T. N. A. Abdelhameed, "Influence of slip condition on unsteady free convection flow of viscous fluid with ramped wall temperature," in Abstract and Applied Analysisvol. 2015, Hindawi, Article ID 327975, 7 pages, Hindawi, 2015. 
[29] N. Marneni, S. Tippa, and R. Pendyala, "Ramp temperature and Dufour effects on transient MHD natural convection flow past an infinite vertical plate in a porous medium," The European Physical Journal Plus, vol. 130, no. 12, p. 251, 2015.

[30] O. A. Beg, M. Ferdows, M. E. Karim et al., "Computation of non-isothermal thermo-convective micropolar fluid dynamics in a Hall MHD generator system with non-linear distending wall," International Journal of Algorithms, Computing and Mathematics, vol. 6, no. 2, pp. 1-44, 2020.

[31] S. Foroushani, D. Naylor, and J. L. Wright, "Heat transfer correlations for laminar free convection in vertical channels with asymmetrically heated isothermal walls," Heat Transfer Engineering, vol. 41, no. 5, 2019.

[32] S. A. Gaffar, V. R. Prasad, O. A. Bég, M. H. Khan, and M. Venkatadri, "Effects of ramped wall temperature and concentration on viscoelastic Jeffrey's fluid flows from a vertical permeable cone," Journal of the Brazilian Society of Mechanical Sciences and Engineering, vol. 40, no. 9, p. 441, 2018.

[33] K. V. B. Rajakumar, M. Umasankara Reddy, K. S. Balamurugan, and K. V. B. S. Raja Ram, "Steady MHD Casson Ohmic heating and viscous dissipative fluid flow past an infinite vertical porous plate in the presence of soret, hall, and ion-slip current," Heat Transfer, vol. 49, no. 3, pp. 1583-1612, 2020.

[34] A. Zaib, U. Khan, A. Wakif, and M. Zaydan, "Numerical Entropic Analysis of Mixed MHD convective flows from a non-isothermal vertical flat plate for radiative tangent hyperbolic blood biofluids conveying magnetite ferroparticles: dual similarity solutions," Arabian Journal for Science and Engineering, vol. 45, pp. 1-20, 2020.

[35] J. C. Maxwell, A Treatise on Electricity and Magnetism, Clarendon, Oxford, UK, 1881.

[36] S. U. Choi and J. A. Eastman, "Enhancing thermal conductivity of fluids with nanoparticles," in Proceedings of 1995 International Mechanical Engineering Congress And Exhibition, San Francisco, CA, USA, November 1995.

[37] F. Ali, M. Saqib, I. Khan, and N. Ahmad Sheikh, "Heat transfer analysis in ethylene glycol based molybdenum disulfide generalized nanofluid via atangana-baleanu fractional derivative approach," in Fractional Derivatives with MittagLeffler Kernel, pp. 217-233, Springer, Cham, Switzerland, 2019.

[38] F. Ali, S. Murtaza, N. A. Sheikh, and I. Khan, "Heat transfer analysis of generalized Jeffery nanofluid in a rotating frame: atangana-Balaenu and Caputo-Fabrizio fractional models," Chaos, Solitons \& Fractals, vol. 129, pp. 1-15, 2019.

[39] Z. Bojarska, J. Kopytowski, M. Mazurkiewicz-Pawlicka et al., "Molybdenum disulfide-based hybrid materials as new types of oil additives with enhanced tribological and rheological properties," Tribology International, vol. 160, Article ID 106999, 2021.

[40] A. Wu, X. Zhao, J. Wang et al., "Application of solid-phase extraction based on magnetic nanoparticle adsorbents for the analysis of selected persistent organic pollutants in environmental water: a review of recent advances," Critical Reviews in Environmental Science and Technology, vol. 51, no. 1, pp. 44-112, 2021

[41] R. Jothi Ramalingam, P. Arunachalam, J. N. Appaturai et al., "Facile sonochemical synthesis of nanoparticle modified Bi$\mathrm{MnOx}$ and $\mathrm{Fe} 3 \mathrm{O} 4$ deposited $\mathrm{Bi}-\mathrm{MnOx}$ nanocomposites for sensor and pollutant degradation application," Journal of Alloys and Compounds, vol. 859, Article ID 158263, 2021.
[42] P. K. Devan, S. Gopinath, K. Rajesh, and S. Madhu, "Improving the characteristics of engine oil using nanofluid as coolant in combat vehicles," Materials Today: Proceedings, vol. 22, pp. 1130-1134, 2020.

[43] F. Ali, Z. Ahmad, M. Arif, I. Khan, and K. S. Nisar, "A time fractional model of generalized Couette flow of couple stress nanofluid with heat and mass transfer: applications in engine oil," IEEE Access, vol. 8, pp. 146944-146966, 2020.

[44] C. R. Kwang-Hua, "Temperature-dependent negative friction coefficients in superlubric molybdenum disulfide thin films," Journal of Physics and Chemistry of Solids, vol. 143, Article ID 109526, 2020.

[45] M. Sgroi, F. Gili, D. Mangherini et al., "Friction reduction benefits in valve-train system using IF-MoS2Added engine oil," Tribology Transactions, vol. 58, no. 2, pp. 207-214, 2015.

[46] M. Turkyilmazoglu, "Natural convective flow of nanofluids past a radiative and impulsive vertical plate," Journal of Aerospace Engineering, vol. 29, no. 6, Article ID 04016049, 2016.

[47] A. T. Akinshilo, J. O. Olofinkua, and O. Olaye, "Flow and heat transfer analysis of the Sodium Alginate conveying Copper Nanoparticles between two parallel plates," Journal of applied and computational mechanics, vol. 3, no. 4, pp. 258-266, 2017.

[48] M. Turkyilmazoglu, "Suspension of dust particles over a stretchable rotating disk and two-phase heat transfer," International Journal of Multiphase Flow, vol. 127, Article ID 103260, 2020.

[49] A. T. Akinshilo, A. G. Davodi, A. Ilegbusi, and G. M. Sobamowo, "Thermal analysis of radiating film flow of sodium alginate using MWCNT nanoparticles," Journal of Applied and Computational Mechanics, (Articles in Press), 2020.

[50] M. Turkyilmazoglu, "Nanoliquid film flow due to a moving substrate and heat transfer," The European Physical Journal Plus, vol. 135, no. 10, pp. 1-13, 2020.

[51] A. T. Akinshilo and G. M. Sobamowo, "Perturbation solutions for the study of MHD blood as a third grade nanofluid transporting gold nanoparticles through a porous channel," Journal of Applied and computational mechanics, vol. 3, no. 2, pp. 103-113, 2017. 